Relations industrielles

Industrial Relations

\title{
Liste des thèses de maîtrise du Département des relations industrielles de l'Université Laval et de l'École des relations industrielles de l’Université de Montréal (1940-1980)
}

Volume 36, numéro 2, 1981

URI : https://id.erudit.org/iderudit/029164ar

DOI : https://doi.org/10.7202/029164ar

Aller au sommaire du numéro

Éditeur(s)

Département des relations industrielles de l'Université Laval

ISSN

0034-379X (imprimé)

1703-8138 (numérique)

Découvrir la revue

Citer ce document

(1981). Liste des thèses de maîtrise du Département des relations industrielles de l'Université Laval et de l'École des relations industrielles de l'Université de Montréal (1940-1980). Relations industrielles / Industrial Relations, 36(2),

435-448. https://doi.org/10.7202/029164ar

Tous droits réservés @ Département des relations industrielles de l'Université Laval, 1981
Ce document est protégé par la loi sur le droit d'auteur. L'utilisation des services d'Érudit (y compris la reproduction) est assujettie à sa politique d'utilisation que vous pouvez consulter en ligne.

https://apropos.erudit.org/fr/usagers/politique-dutilisation/ 


\section{Thèses de maîtrise en relations industrielles}

\section{Université Laval et Université de Montréal: 1940-1980}

\section{Thèses de maîtrise en relations industrielles à l'Université Laval}

Toutes ces thèses peuvent être consultées à la Bibliothèque générale de l'Université Laval.

AIRD, Roger, Rapports entre l'action catholique et l'organisation professionnelle ouvrière et patronale d'inspiration chrétienne, $1952,326 \mathrm{pp}$.

ANGER, Bernard, Syndicalisme et fonctionnarisme, 1961, $31 \mathrm{pp}$.

ARCHAMBAULT, Jacques, Comités d'entreprises et Comités-mixtes de production, 1948, $112 \mathrm{pp}$.

ASSELIN, Roger, Le statut juridique de l'association professionnelle, patronale et ouvrière dans la province de Québec, 1954, $125 \mathrm{pp}$.

BEAUDOIN, Guy, La grève chez Price Brothers \& Company Limited en 1943, 1957, 159 pp.

BEAULIEU, Jean-Claude, Concepts théoriques et syndicat, 1960, $141 \mathrm{pp}$.

BÉLANGER, Charles, Les régimes de congés payés dans le Québec, 1950, 317 pp. BÉLANGER, Jacques, La négociation collective entre la Commission de transport de la communauté urbaine de Montréal (CTM-CTCUM) et le Syndicat des employés de transport de Montréal (CSN): 1964-1973, 1978, 228 pp.

BÉLANGER, Laurent, La référence aux valeurs dans les relations patronalesouvrières, 1961, $106 \mathrm{pp}$.

BÉLANGER, Oliva, La formation professionnelle dans les centres d'apprentissage, 1949, 229 pp.

BERNARD, Michel, Réévaluation des clauses d'ancienneté face aux changements industriels, 1970, $164 \mathrm{pp}$.

BERNIER, Jean, Législation du travail concernant les corporations municipales et scolaires et leurs employés. La Loi et la jurisprudence, 1964, 132 pp.

BERNIER, Paul-Étienne, Essai sur la convention collective, 1944, 77 pp.

BILODEAU, Pierre-Paul, L'unité syndicale au Québec 1880-1921, 1979, 222 pp.

BLAIS, Fernand, La mobilité occupationnelle des cadres supérieurs en relations industrielles dans l'entreprise au Québec, 1969, 133 pp.

BLAIS, Ginette, Culture et productivité du travail, 1973, $88 \mathrm{pp}$.

BLAIS, Michel, La réorganisation d'un service de personnel: étude de cas, 1969, 178 pp.

* La présente compilation inclut les titres déjà publiés dans la liste annotée des thèses de maîtrises en relations industrielles, Université Laval et Université de Montréal, publiée dans Relations industrielles, vol. 21, no 4 (1966), pp. 629-655 et la liste publiée dans Relations industrielles, vol. 23, no 4 (1968), pp. 684-685.

** La compilation de cette liste a été complétée par Claudine Leclerc, Département des relations industrielles, Université Laval.

*** La liste des thèses déposées à l'Université de Montréal et inclue dans la présente compilation a été fournie par l'École de relations industrielles de l'Université de Montréal. 
BLAIS, René, La Commission d'enquête sur l'exercice de la liberté syndicale dans l'industrie de la construction: la Commission Cliche, 1979, $256 \mathrm{pp}$.

BOIVIN, Jean Le syndicalisme chez les professionnels du gouvernement du Québec, 1968, 191 pp.

BOUCHARD, Paul, Étude comparative des législations ou mesures gouvernementales actuelles en matière de formation professionnelle des travailleurs et des sanstravail, 1967, $166 \mathrm{pp}$.

BOUDRIAS, Gervais, Étude de cas sur le journal d'usine au Québec, 1967, 76 pp. BOUTIN, Gilles, Pénurie ou surplus de médecins au Canada, 1974, 189 pp.

BRIEN, Pierre, Administration du personnel et marketing, 1969, $218 \mathrm{pp}$.

BRISSON, Chantal, L'utilisation de la convention collective en tant qu'outil d'intervention dans le domaine de la santé au travail, 1980, 166 pp.

CABOT, Roger, Le conflit de reconnaissance syndicale de Murdochville en 1957, $1966,147 \mathrm{pp}$.

CANTIN, Jean-Mathieu, Une enquête sur un journal d'entreprise, 1947, 157 pp.

CARON, Martin, Situation économique et sociologique des propriétaires et marins de goélettes, 1960, $90 \mathrm{pp}$.

CHANDONNET, Jean, Le problème de la syndicalisation dans le commerce au détail de Québec, 1956, $52 \mathrm{pp}$.

CHARTIER, Paul, Les normes d'apprentissage dans l'industrie du bâtiment, 1950, $86 \mathrm{pp}$.

CHARTIER, Roger, Collaboration patronale-ouvrière chez les barbiers et coiffeurs (région de Québec), 1950, $156 \mathrm{pp}$.

CIMON, Paul, La participation de représentants de syndicats locaux à un conseil syndical local, 1966, $166 \mathrm{pp}$.

CORRIVEAU, Laurent-A., Étude sur les relations industrielles au mouiin Anglo canadien de pulpe et de papier, 1945, $34 \mathrm{pp}$.

COSSETTE, Jean, Comités de collaboration patronale-syndicale: étude de cas, 1967, $153 \mathrm{pp}$.

COUTURE, Jacquelin, L'assurance-hospitalisation et le travailleur hospitalier, 1965, $97 \mathrm{pp}$.

CRÉPIN, Pierre, Le patronat québécois et la planification économique, 1967, 114 pp.

CROTEAU, Majella, Étude de l'évolution du décret et de l'industrie du meuble dans la province de Québec de 1935 à 1955, 1956, $80 \mathrm{pp}$.

DE LA CHEVROTIÈRE, Jacques, Les comités mixtes de production, 194.5, 65 pp. DESBIENS, Robert, Inventaire des effectifs humains travaillant dans les établissements hôteliers du Québec métropolitain, 1977, 167 pp.

DESCHÊNES, Guy, Généralités sur l'évaluation des emplois, 1955, 80 pp.

DESCHÊNES, Jean-Paul, Wage-Incentive Systems, 1958, $101 \mathrm{pp}$.

DESGAGNÉS, Georges, Implications économiques d'une réduction des heures de travail, 1967, $89 \mathrm{pp}$.

DESPRÉS, Jean-Pierre, Le Canada et l'Organisation internationale du Travail, 1946, 372 pp.

DION, Denys, Étude comparative des lois des syndicats professionnels de France et de Québec, 1949, 153 pp. 
DIONNE, Pierre, Une analyse historique de la Corporation des enseignants du Québec, (1836-1968), 1969, 260 pp.

DROUIN-LAPOINTE, Denise, Les travailleurs de la compagnie Rolland et quelques aspects de la vie économique et du travail. Sondages d'opinions et d'attitudes, 1962, $104 \mathrm{pp}$.

DUMAS, Pierre, Les services publics et la grève, 1965, $75 \mathrm{pp}$.

DUPUIS, Roger B., Collective Bargaining in the Provincial Public Service Based on the Saskatchewan Experience, 1967, 104 pp.

FERLAND, Jean-Paul, Analyse comparative des conventions collectives dans l'industrie de la pulpe et du papier de la province de Québec, en 1945, 1947, 74 pp.

FORGUE, Claude, Le plan conjoint des producteurs de lait industriel du Québec, $1971,187 \mathrm{pp}$.

GAGNÉ, Jean, Monographie du service du personnel de la Montreal Cottons Limited, Salaberry-de-Valleyfield, Québec, 1948, $217 \mathrm{pp}$.

GAGNON, Gilbert, Les régimes de participation au profit aux États-Unis, 1966, 129 pp.

GARNEAU, Charles-Édouard, Conciliation et arbitrage dans les conflits du travail, 1942, $38 \mathrm{pp}$.

GAUDREAU, Claude, La réglementation des conditions de travail dans les services de l'automobile (région de Québec), 1953, 217 pp.

GIRARD, Michel, La santé et la productivité du travail, 1973, $111 \mathrm{pp}$.

GOSSELIN, Hélène, Signification des activités de travail (professionnelles) et hors travail (extra-professionnelles) pour des femmes occupant certains postes de professionnelles à la Fonction publique du Québec, 1978, 155 pp.

GOSSELIN, Jean-Yves, Décret relatif à l'industrie de la boulangerie - arrêté ministériel no 659 du 13 mai 1948, 1949, 124 pp.

GOSSELIN, Louis, La fonction personnel dans l'entreprise québécoise, aspect global et aspects particuliers: les perceptions que les directeurs généraux et les directeurs du personnel s'en font, 1979, $179 \mathrm{pp}$.

GOSSELIN, Louis-de-Gonzague, Intervention supplétive de l'État par la fixation du salaire minimum dans le Québec, $1950,77 \mathrm{pp}$.

GOULET, Denis, Évaluation des structures de négociation dans les secteurs des hôpitaux et de l'enseignement public au Québec: 1964-1972, 1975, 169 pp.

GROSS, François, Propriété et autorité dans l'entreprise moderne, 1965, 81 pp.

GUILBAULT, Jacques, Loi provinciale de conciliation et d'arbitrage, 1950, $165 \mathrm{pp}$.

GUY, Paul, Essai sur les relations du travail dans le secteur de l'enseignement public élémentaire et secondaire au Québec, 1970, 198 pp.

HARDY, Guy-Paul, Analyse de l'évolution des clauses d'ancienneté dans quelques grands établissements manufacturiers québécois, 1965, $124 \mathrm{pp}$.

HOUDE, Eugène, Le droit de grève dans les services publics, 1964, 163 pp.

JOBIN, Gilles, Les problèmes administratifs dans le système coopératif, 1966, 115 pp.

LABELLE, Rhéal, Monographie industrielle de la compagnie E.B. Eddy, Hull, 1953, $177 \mathrm{p}$.

LABRANCHE, Raymond, Relations publiques de l'entreprise. Fonction et département, 1964, 129 pp. 
LADOUCEUR, Jean, Planification de la main-d'oeuvre au niveau de la firme, 1975, $243 \mathrm{pp}$.

LAFLAMME, Gilles, Éducation syndicale à la Confédération des syndicats nationaux, 1968, $125 \mathrm{pp}$.

LAFLAMME, Marcel, L'approche diagnostique globale en développement organisationnel, 1976, $331 \mathrm{pp}$.

LALANDE, Serge, La Commission des relations de travail et la détermination de l'unité de négociation, 1966, $106 \mathrm{pp}$.

LANGEVIN, Jean-Louis, Une nouvelle approche à la gestion des ressources humaines, la direction participative par objectifs: concepts et exercices d'apprentissage, 1976, 273 pp.

LARIVIÈRE, Robert, Les procédures d'organisation du personnel dans la Fonction publique provinciale (1967), 1972, $140 \mathrm{pp}$.

LAVIGNE, Georges-A., Le grief. La clause et la procédure de règlement des griefs dans les conventions collectives, 1947, $136 \mathrm{pp}$.

LAVOIE, Gilles, Les pratiques déloyales dans les conflits ouvriers, États-Unis, Canada, Québec, 1945, 81 pp.

LÉCUYER, Normand, Société de reclassement des travailleurs de l'amiante (1964): trois ans après, 1970, $156 \mathrm{pp}$.

LEFEBVRE, Gaston, Structure sociale de l'entreprise, 1953, 147 pp.

LEVESQUE, Gérard-Majella, Heures de travail, vacances payées, jours fériés et rémunération de surtemps dans certains décrets de la province de Québec, 1956, 141 pp.

LEVESQUE, Richard, Le règlement des griefs dans la Fonction publique du Québec, 1970.

L'HEUREUX, Gilbert, La sécurité d'emploi du travailleur face à l'auiomation, 1965, 95 pp.

LIZOTTE, Jean, Monographie d'un service du personnel dans un magasin à rayons, $1949,180 \mathrm{pp}$.

LORTIE, Guy, L'évolution de l'action politique de la Confédération des syndicats nationaux, 1965, $98 \mathrm{pp}$.

MARCHAND, Jean, Nature et sources du socialisme, 1942, $61 \mathrm{pp}$.

MARIER, Roger, L'assurance sociale est fonction de la prudence politique, 1941, 74 pp.

MARLEAU, Marcel, La rotation de la main-d'oeuvre à la Montreal Cottons Limited, Salaberry-de-Valleyfield, Québec, 1950, $101 \mathrm{pp}$.

MARQUIS, Léopold, L'utilisation des travailleurs âgés, 1955, 87 pp.

MERCIER, Jacques, La procédure de préavis de licenciement et l'article 45 du chapitre 51 des lois de 1969 du Québec, 1975, 214 pp.

MERCURE, Arthur, Analyse des tâches au Syndicat de Québec Ltée, 1963, 69 pp. MOISAN, André, L'apprentissage des métiers de la construction dans la région de Québec, 1952, $151 \mathrm{pp}$.

MONETTE-GUY, Réjeane, Essai sur l'éducation de la main-d'oeuvre, 1973, 136 pp. MONTPETIT, Jean-Guy, La préférence syndicale dans l'industrie de la construction (Région de Québec), 1961, 66 pp.

MOREAU, Lionel, Les relations employeurs-employés dans les services hospitaliers, région de Québec, 1932-1951, 1951, 215 pp. 
NÉRON, Gilles, Étude sur quelques aspects de l'amendement du 12 septembre 1963 au décret 3003, relatif à l'industrie de la chaussure, 1964, 184 pp.

ODSTRCIL, Vladimir, Exploitation by Integration, 1955, $150 \mathrm{pp}$.

OUELLET, Gaston, Influence de l'âge et de l'instruction sur les groupes de travail, 1967, $131 \mathrm{pp}$.

OUELLET, Jacques, Fonctions économiques et sociales de l'entreprise Canadian General Electric, Québec, 1952, 86 pp.

PARENT, André, Ce que pensent les employeurs du Québec qui ont expérimenté les clauses de sécurité syndicale, 1947, 62 pp.

PARENT, Serge, Le mouvement corporatiste québécois, 1967, 116 pp.

PARENTEAU, René L., L'évolution du nationalisme à la CTCC-CSN, 1970, 307 pp.

PARISÉ, Robert, Grève dans la construction à Baie-Comeau en 1957, 1960, 89 pp. PÉPIN, Marcel, Monographie syndicale de la Fédération nationale catholique du Textile, 1949, $119 \mathrm{pp}$.

PETIT, André, La formation professionnelle des adultes: historique et évolution de l'expérience québécoise dans le contexte canadien, 1972, $408 \mathrm{pp}$.

PLEAU, Ronald, Syndicalisme chez les ingénieurs, succès ou échec?, 1966, 258 pp. POIRIER, Charles-Édouard, Études sur le décret relatif au commerce de l'alimentation au détail, 1949, $97 \mathrm{pp}$.

POULIN, Gabriel, Fluctuations du nombre annuel des accidents dans une entreprise de transport en commun, 1957, $71 \mathrm{pp}$.

POULIN, Marc, La corruption syndicale, 1966, $87 \mathrm{pp}$.

POULIOT, Guy, Le statut de l'assureur-vie au Québec, 1970, 361 pp.

PRÉCOURT, Alain, L'Unité syndicale au Québec: 1940-1960, 1981, 190 pp.

RHEAULT, Jean-Paul, De la politique de main-d'oeuvre, 1967, 105 pp.

RHEAULT, René, Sécularisation et syndicalisme, 1967, 143 pp.

RICARD, Louise, Monographie industrielle du Centre médical de la Cie Dominion Corset à Québec, 1949, $87 \mathrm{pp}$.

RICHARD, Thomas, Le syndicalisme ouvrier d'après-guerre en Italie, 1950, $131 \mathrm{pp}$. ROY, Rosaire, Position et préoccupation de la Confédération des syndicats nationaux et de la Fédération des travailleurs du Québec sur le travail féminin, 1968, 162 pp.

SAINT-CYR, Michel, L'administration du personnel et sa condition dans les entreprises de la ville de Québec, 1955, $107 \mathrm{pp}$.

SAINT-HILAIRE, Lucien, L'utilisation rationnelle de la main-d'oeuvre, 1955, 102 pp.

SAMSON, Roger, Syndicalisme et progrès technique, 1966, $87 \mathrm{pp}$.

SARKIS, M. Bassam, Le système de relations industrielles au Liban, 1976, 166 pp. SAUVAGEAU, Guy, Identification à l'organisation dans le secteur public, parapublic et privé au Québec, 1980, 152 pp.

SAVARD, André, Essai de définition et de calcul du roulement de la main-d'oeuvre, 1957, 185 pp.

SEXTON, Jean, La CTCC-CSN: du corporatisme à la réforme de l'entreprise, 1970, 178 pp. 
SINCENNES, Aldège, Le décret du commerce de gros de l'alimentation dans la région de Québec, 1949, $146 \mathrm{pp}$.

TALBOT, Pierre, Le syndicalisme des fonctionnaires municipaux, 1955, $159 \mathrm{pp}$.

TÊTU, Claude, L'histoire orale et les relations industrielles, 1967, $135 \mathrm{pp}$.

THÉRIEN, Charles-Édouard, Relations patronales-ouvrières dans l'industrie de la chaussure de la province de Québec, 1949, $182 \mathrm{pp}$.

TORRES, Frederico, Estudio de un complejo industrial, 1965, 248 pp.

TOURIGNY, Pierre, Les facteurs influençant le port des protecteurs auditifs dans trois industries du meuble de la région de Laurier Station au Québec, 1980, 118 pp. TOUSIGNANT, Fabienne, Étude comparative des salaires dans l'indusirie textilesoie et rayonne de la province de Québec, 1950, 108 pp.

TREMBLAY, Florent, Conflit des tendances et problème de pouvoir dans le syndicalisme français de l'entre-deux guerres, 1971, $114 \mathrm{pp}$.

TREMBLAY, Laurent, Implication de la mécanisation sur certaines caractéristiques $d u$ secteur forestier, 1966, $163 \mathrm{pp}$.

TREMBLAY, Louis-Marie, Le marché du travail dans la région de Québec, 1957, $120 \mathrm{pp}$.

TREMBLAY, Marcel, L'industrie de la chaussure du Québec et son problème de formation professionnelle, 1953, $279 \mathrm{pp}$.

TREMBLAY (BRASSARD), Raynald, Sur la publicité de l'Aluminium Company of Canada pendant la grève d'Arvida en 1957, 1961, 176 pp.

VAILLANCOURT, Mathieu, La distribution des pouvoirs à la Confédération des Syndicats nationaux, 1966, $77 \mathrm{pp}$.

VALLÉE, Émile, Les unions internationales et la concurrence des syndicats confessionnels au Québec, 1966, 118 pp.

VO THU, Nguyet, La convention collective et l'adaptation de la main-d'oeuvre hospitalière aux changements, 1973,

YACCARINI, Benoît, Les tribunaux du travail, 1949, 130 pp.

\section{Thèses de maîtrise en relations industrielles à l'Université de Montréal}

AGLOSSI, Emmanuel, Syndicalisme et problèmes de développement au Dahomey, 1972, 198 pp.

ARCHAMBAULT, Guy, Analyse des différentiations de salaires, Québec-Ontario, 1955, $147 \mathrm{pp}$.

ARES, Jean-Claude, L'industrie de l'Aluminium au Canada,

AUGER, René, L'échantillonnage dans la statistique de la main-d'oeuvre, 1948, 57 pp.

BEAUDET, Colette, Fonctions nouvelles à l'intérieur d'un pays, 1952, 2.29 pp.

BEAUDOIN, Francine, L'influence du taux de salariat sur la part des salaires dans le revenu national, $1961,83 \mathrm{pp}$.

BEAUGRAND-CHAMPAGNE, Guy, Le logement à Montréal des étudiants Forains à l'université de Montréal et son incidence au point de vue social sur la vie étudiante, 1948, $115 \mathrm{pp}$. 
BÉDARD, Roger, La pensée de $M$. Slighter sur le régime de la convention collective, 1954, $140 \mathrm{pp}$.

BÉLANGER, Claude, Évolution des droits de la direction, 1955, $118 \mathrm{pp}$.

BENGLE, Normand, Application de l'approche systémique en relations industrielles, 1977, $166 \mathrm{pp}$.

BERGERON, Jean-Louis, L'utilisation d'un système de notation du personnel en relation avec l'administration des salaires: étude de cas, 1962, $77 \mathrm{pp}$.

BERNATCHEZ, Jean-Claude, Étude de facteurs explicatifs d'absentéisme dans un centre hospitalier, 1977, $159 \mathrm{pp}$.

BESNER, Jacques, Diagnostic en gestion du personnel. Proposition d'un instrument, 1980,

BIBEAULT, Réal, Le syndicat des débardeurs de Montréal, 1954, 63 pp.

BIRON, Alice, Étude du service social de groupe, 1948, 55 pp.

BISAILLON, Robert, La sélection des chefs dans le service militaire, 1953, 108 pp.

BLAIS, Jean, Les finances publiques au Canada,

BLAIS, Raynald, L'idéologie économique de la confédération des syndicats nationaux, 1971, $272 \mathrm{pp}$.

BOILY, Jean, La conciliation au Québec: un système nouveau et plus efficace, 1968, 239 pp.

BOLDUC, Yvon, Organisations ouvrières et droit syndical fédéral, 1952, 162 pp.

BOUVIER, Sylvio, Le revenu familial, 1946, $62 \mathrm{pp}$.

BRIZARD, Louis-Philippe, Étude théorique des facteurs déterminant la localisation industrielle et leur application à l'industrie de la chaussure de Québec, 1948, 95 pp.

BROSSARD, Michel, L'idéologie économique de la FTQ, 1969, 347 pp.

CAMPEAU, Aimé, Administration du personnel à l'Hydro-Québec, 1957, 85 pp.

CARBONNEAU, Roger, Sécurité industrielle, 1958, $121 \mathrm{pp}$.

CARDIN, Jean-Réal, Syndicalisme national catholique et droit syndical québécois, $1948,125 \mathrm{pp}$.

CARR RIBEIRO, Roberto, Certaines caractéristiques des employés qui participent au plan de suggestions dans une usine de Montréal, 1968, 109 pp.

CARON, Louise, Le travail féminin dans la province de Québec, 1955, $98 \mathrm{pp}$.

CAUCHON, Léon, L'apprentissage dans l'industrie de la construction (1945-1952), 1952, $120 \mathrm{pp}$.

CHAGNON, André, La fonction de gestion du personnel dans la petite entreprise manufacturière, 1959, $228 \mathrm{pp}$.

CHAOUCHE-RAMDANE, Djemale, La formation, instrument d'adaptation au changement: l'expérience algérienne de formation (cas de la Sonatrach), 1978, 271 pp.

CHARBONNEAU, Jean, Organisation des employés de bureau, 1954, 65 pp.

CHARBONNEAU, Réal, Maisons ouvrières et sécurité économique,

CHARPENTIER, Rolland, Basis for the Classification of Salaried and Hourly Paid Employees, 1960, $87 \mathrm{pp}$.

CHESNAY, Jean, Permanents syndicaux et professionnalisme, 1979, 145 pp.

CHEVRIER, Micheline, Une paroisse ouvrière en formation, 1948, $53 \mathrm{pp}$.

CHOINIÈRE, Monique, Le travail industriel féminin à domicile, 1946, $57 \mathrm{pp}$. 
CHOQUETTE, Robert, La sélection des agents d'assurance par l'étude des données biographiques que l'on retrouve sur la fiche de demande d'emploi, 1972, 169 pp.

CINQ-MARS, Normand, Les influences du syndicalisme sur le niveau des salaires, 1966, 147 pp.

CLÉMENT, Antonio, La détention du pouvoir économique dans la grande entreprise, $1960,115 \mathrm{pp}$.

CLÉROUX, Michel, Le leadership chez John L. Lewis, 1961, 159 pp.

COLLARD, J.M., Les communicatios vers le haut dans l'entreprise, 1962, $122 \mathrm{pp}$. CONTI, Raymond, Les relations syndicales canado-américaines, 1959, $165 \mathrm{pp}$.

CÔTÉ, Alphonse, Une agglomération polono-ukrainienne de Montréal, 1948, 90 pp.

COUPAL, Michel, Étude de quelques variables individuelles et situationnelles différenciant les directeurs de succursales les plus efficaces et les moins efficaces, 1970, 385 pp.

COURVILLE, Claude, L'arbitrage obligatoire, 1954, $100 \mathrm{pp}$.

CRÉPEAU, Marcel, La garantie annuelle de salaire, 1950, $119 \mathrm{pp}$.

CRÉPEAU, Marcel, La détermination des critères de rémunération pour une ville d'importance, 1972, $224 \mathrm{pp}$.

CURZI, Paul, Les relations ouvrières-patronales dans le secteur hospitalier à Montréal, 1961, 137 pp.

DAGENAIS, Michel, Le hasard, la tendance individuelle et l'accidentabilité comme facteur d'explication des accidents du travail, 1972, $129 \mathrm{pp}$.

DAIGNEAU, Marius, Les prérogatives de la gestion et la convention collective, 1961, $108 \mathrm{pp}$.

DALLAIRE, Gérard, Les relations entre patrons et ouvriers de la forêt du Québec, 1952, $107 \mathrm{pp}$.

DALLAIRE, Germain, Les conditions de travail dans l'industrie de la construction au Canada, 1960, 74 pp.

DANDONNEAU, Normand, La grève des enseignants de l'Estrie, 1965, 158 pp.

DAOUST, Claude, Les ingénieurs et le syndicalisme dans la province de Québec, 1965, 198 pp.

DÉCARY, Marielle, La sélection du personnel, 1951, $111 \mathrm{pp}$.

DE GUISE, J.-Guy, Monographie historique du Conseil central des syndicats nationaux de Montréal de 1920 à 1955, 1962, 160 pp.

DELISLE, Jean-Paul, Différenciation des salaires masculins et féminins, 1949, 89 pp.

DELISLE, Léo, L'organisation de l'apprentissage dans la province de Québec, 1955 , $105 \mathrm{pp}$.

DELORIMIER, François, Les grèves de Sorel de 1937, 80 pp.

DELORME, François, Étude empirique de la satisfaction au travail chez les contremaîtres québécois, 1975, 324 pp.

DEOM, André, La grève de Lachute, 1951, 111 pp.

DEPORQ, Jean-Marie, Les structures et pouvoirs de la confédération des syndicats nationaux, 1970, $150 \mathrm{pp}$.

DESCHÊNES, Jean-Claude, La formation des cadres, 1959, 264 pp.

DÉSILETS, Marcel, Immigration au Canada 1945-1951, 1953, 88 pp. 
DESLIERRES, Jean-Paul, L'agent de liaison dans l'industrie, 1946, $73 \mathrm{pp}$.

DJANDJI, Guy N., Analyse de la théorie d'Herzberg face à la rémunération, 1973, $134 \mathrm{pp}$.

DUBOIS, Émile, Les coopératives d'habitation de la province de Québec, 1949, 72 pp.

DUCHESNE, Rosaire, Facteurs déterminants de l'émigration des ingénieurs originaires du Saguenay-Lac St-Jean, 1974, 285 pp.

DUFOUR, Ghislain, L'atelier syndical, 1958, $115 \mathrm{pp}$.

DUGUAY, Pierre, La formule de demande d'emploi dans la sélection du personnel, $1971,182 \mathrm{pp}$.

DULUDE, André, Politiques comparées des syndicats de journalistes de quelques quotidiens du Québec et du Canada anglais, 1978, 356 pp.

DUMAIS, André, Procédure et réglementation des griefs et incidences sur le régime de la convention collective, 1954, $112 \mathrm{pp}$.

DUBERRY, Fernand, Tarification ferroviaire et rendement social, 1950,

DUPÉRÉ, Yves, La méthode de comparaison des facteurs dans l'évaluation des emplois, 1963, $110 \mathrm{pp}$.

DUPRAS, Pierre, Le service du personnel dans l'entreprise moderne, 1961, $134 \mathrm{pp}$. DUPRÉ, Alphonse, Relations patronales-ouvrières entre CECM et APCM, 1968, $362 \mathrm{pp}$.

DUROCHER, Gilles, Experience Rating ou Système de classement au mérite, 1956, 87 pp.

DUROCHER, Lionel, La formation des contremaîtres, 1952, 73 pp.

ÉTHIER, Jean-Marie, Le coût de la vie à Asbestos en 1945, 1946, 108 pp.

FERLAND, Gilles, La politique salariale dans le secteur public québécois: une comparaison secteur public, secteur exposé, 1979, 143 pp.

FERRAGNE, Roger, Le syndicalisme dans les hôpitaux catholiques de Montréal, 1952, $190 \mathrm{pp}$.

FORGET, Marcel, Contribution à une monographie de St-Sylvère: évolution économique de St-Sylvère 1925-1945, 1947, 76 pp.

FORTIER, Paul-Émile, Efficacité et efficience d'une organisation, 1966, 122 pp.

FORTIER, Paul-Émile, L'état d'équilibre de l'entreprise, 1966, 187 pp.

FORTIN, Bruno, Détermination des salaires dans l'industrie du pain dans l'île de Montréal,

FORTIN, Maurice, L'influence de la substitution de nature technique sur la fonction qualitative du facteur «Travail», 1967, $119 \mathrm{pp}$.

FOURNIER, Léonard, Climats des relations de travail au début du siècle dans la province de Québec, 1956, $220 \mathrm{pp}$.

GAGNON, Jean-Yves, La direction du personnel dans la fonction publique fédérale canadienne: le partage d'autorité, 1967, $177 \mathrm{pp}$.

GAGNON, Mona-Josée, La femme dans l'idéologie québécoise et dans la CSN: étude idéologique et monographie syndicale, 1973, $281 \mathrm{pp}$.

GARCIA PENA, Aurelio, L'action politique syndicale au Vénézuéla, 1968, 148 pp. GAREAU, Maurice, Étude des critères d'avancement professionnel, 1955, $82 \mathrm{pp}$.

GAREAU, Raphaël, Les variations du chômage dans la province de Québec, 1956, $50 \mathrm{pp}$. 
GAUDET, Hélène, Analyse du marché du travail des étudiants (région de Montréal, 1969), 1971, 145 pp.

GAUDREAU, Raymond, Innovation, emploi et salaire, 1949, 85 pp.

GAUTHIER, Guy, Analyse critique du Conseil national mixte du service public du Canada, 1975, 144 pp.

GAUTHIER, Jacques, Analyse critique de la loi sur la formation et la qualification professionnelles de la main-d'oeuvre: solution aux problèmes de la formation professionnelle au Québec, 1972, $128 \mathrm{pp}$.

GAUTHIER, Jean, L'éducation ouvrière dans la région de Montréal, 1955, $92 \mathrm{pp}$. GENDRON, Francine, La protection individuelle et collective du droit d'association, $1978,162 \mathrm{pp}$.

GERMAIN, Denis, Le salaire annuel garanti, 1955, $204 \mathrm{pp}$.

GINGRAS, Albert, Évaluation des fonctions d'écriture à la ville de Montréal selon une technique nouvelle (1949), 1950, $360 \mathrm{pp}$.

GIRARD, P., La négociation en front commun syndical au printemps 1966 dans l'industrie de la construction à Montréal, 1970, $271 \mathrm{pp}$.

GOSSELIN, Pierre, La prévention des accidents et l'utilisation des données biographiques à l'embauchage, 1972, $147 \mathrm{pp}$.

GOSSELIN, Roger, Analyse micro-économique de l'automation, 1957, 151 pp.

GOULET, Jean-Guy, Contexte expérimental facilitant la cohésion des groupes de travail: étude de cas, 1973, 157 pp.

GOULET, Michel, La protection du revenu dans les conventions collectives publiques québécoises, 1976, $246 \mathrm{pp}$.

GROULX, Gilles, Le syndicalisme dans l'industrie textile du Québec, 1954, 154 pp. GUÉRIN, Gilles, Modèle mathématique de planification de la main-d'oeuvre, 1968, 146 pp.

HAMEL, Yvon, Le fondement de l'autorité dans l'usine capitaliste, 1953, 104 pp. HÉBERT, Gérard, Collective Bargaining and Labour's Share in National Income, $149 \mathrm{pp}$.

HÉBERT, Gilles, Étude sur la motivation à la formation scolaire, 1966, 155 pp.

HÉBERT, Lucien, Le comité de coopération industrielle, 1946, 55 pp.

HÉBERT, Roger, Le conseil supérieur du travail, 1969, 128 pp.

HENRIPIN, Jacques, L'industrie du coton primaire $d u$ Canada,

HERMAN, Emil Edward, The Problem of Determination of the Appropriate Bargaining Units Labour Relations Boards in Canada, 1965, 374 pp.

HERMANN, E., The Bargaining Unit, 1963, 9 pp.

HUBERT, Pitre, Un service du personnel dans une entreprise à contrat, 1962, $97 \mathrm{pp}$. JOLICOEUR, Maurice, Les collets blancs et le syndicalisme, 1958, 191 pp.

JULIEN, Bruno, Le contrôle des mises à pied dans l'organisation industrielle, 1954, $311 \mathrm{pp}$.

LABONTÉ, André, L'automatisation des bureaux: nouvelles compositions de la force du travail, 1968, $356 \mathrm{pp}$.

LACOSTE, Jean-Guy, Plan de pension à la retraite, 1954, $120 \mathrm{pp}$.

LACOSTE, Lucien, Évaluation des emplois, 1947, 93 pp.

LAFONTAINE, Richard, Étude du régime de pré-retraite en vigueur à l'Aluminium du Canada Ltée, 1973, 159 pp. 
LAFRENIÈRE, René, Étude sur l'égalisation des salaires dans une entreprise à usines multiples, 1958, $107 \mathrm{pp}$.

LALANDE, Jean-Marie, Analyse du marché des ingénieurs au Canada, 1965, 126 pp.

LALONDE, Germain, Étude de la satisfaction au travail chez les cadres d'une entreprise, 1979, $192 \mathrm{pp}$.

LAMARCHE, Hubert, L'étendue, le modèle et les déterminants de la mobilité industrielle des conseillers en relations industrielles de la province de Québec, 1971, 235 pp.

LAPORTE, André, Une étude des systèmes disciplinaires dans l'entreprise moderne, $1960,115 \mathrm{pp}$.

LAPORTE, Pierre, La participation par le processus de la négociation continue: étude de cas, 1978, $212 \mathrm{pp}$.

LARAMÉE, Pierre P., Psychométrie au sein de l'entreprise, 1956, 83 pp.

LAREAU, Raymond, L'influence du style de supervision sur la satisfaction au travail, 1973, $183 \mathrm{pp}$.

LARIVIÈRE, Sylvio, Évolution des salaires, 1955, $133 \mathrm{pp}$.

LAROUCHE, Viateur, L'évolution des unités de négociation dans le secteur hospitalier 1960-1965, 1966, $185 \mathrm{pp}$.

LAUZON, Hubert, Étude monographique sur les fonctions des unions et du mouvement syndical, 1967, $106 \mathrm{pp}$.

LAVOIE, Jean-Marie, L'utilisation efficace de l'ingénieur, 1958, 127 pp.

LECLERC, Louis, La jurisprudence arbitrale québécoise en matière de congédiement, 1978, $356 \mathrm{pp}$.

L'ÉCUYER, Richard, Quelques caractéristiques du leadership chez les infirmières, 1970, $109 \mathrm{pp}$.

LEDOUX, Léo, L'évolution comparée des salaires au Canada et aux États-Unis de 1901 à 1951, 1954, 192 pp.

LEFEBVRE, Lina, L'ouvrière, domiciliaire de Grand'Mère, 1948, $80 \mathrm{pp}$.

LEGAULT, Yvan, Les congés annuels payés dans l'industrie du bâtiment de la province de Québec, 1948, $66 \mathrm{pp}$.

LE LOUARN, Jean-Yves, Étude empirique de la satisfaction au travail des professeurs de l'Université de Montréal, 1978, 138 pp.

LEMIRE, Hubert, Conditions de travail dans les magasins à chaîne de l'épicerie, 1952, $90 \mathrm{pp}$.

LESAGE, Jacques, Le syndicalisme chez les fonctionnaires municipaux de la ville de Montréal, 1957, 73 pp.

LEVERT, Roland, Les plans de suggestion, 1959, 59 pp.

L'HEUREUX, Jean, Étude du profil et de la motivation des travailleurs intéressés à la formation lors du reclassement, 1974, $158 \mathrm{pp}$.

LUCIER, Jacques, La sécurité syndicale, 1953, 129 pp.

LUSSIER, Roger, Le contrôle syndical des renvois et des licenciements, 1946, 66 pp.

MALLETTE, Noël, Le renvoi pour activité syndicale, 1970, 252 pp.

MALO, Fernand, Flexibilité des salaires, 1953, 100 pp.

MALO, Gilles, L'inflation et les salaires, 1961, $76 \mathrm{pp}$.

MALO, Roland, La participation aux bénéfices, 1953, 119 pp. 
MANSEAU, Suzanne, Évaluation des emplois dans une section d'une usine canadienne où l'on fabrique des appareils d'optiques, 1948, 64 pp.

MARION, Gérard, La politique canadienne sur les monopoles, 1957, 187 pp.

MARTIN, Gilles, Bien-être collectif et contrôle du change étranger, 1950.

MARTIN, Jacques, Les chevaliers du travail et le syndicalisme international à Montréal, 1965, $135 \mathrm{pp}$.

MARTIN, Roger, Problème d'ancienneté survenu à la Cie de transport de Montréal, 1953, $122 \mathrm{pp}$.

MATTEAU, Arthur, Étude sur l'émigration des jeunes à St-Sylvère, 1925-1945, 1948, $95 \mathrm{pp}$.

MAURICE, Gérard U., La formation des chefs et l'industrie de la pulpe et du papier du Québec, 1957, 118 pp.

MEJIA, Francisca Javier, The Encyclicals and the Colombian Industrial Worker Problem, 1950, $65 \mathrm{pp}$.

MERCIER, André, Le problème de l'éducation physique dans les universités canadiennes-françaises, 1948, $97 \mathrm{pp}$.

MERCIER, Normand, Les incidences de l'assurance-hospitalisation sur la prise de décision en relations ouvrières dans le secteur hospitalier du Québec, 1967, 116 pp. MICHAUD, Paul, L'assurance-vie de groupe, 1956, $35 \mathrm{pp}$.

MINVILLE, Jean, Le rôle de la "Comparaison» dans la négociation collective, 1957, $110 \mathrm{pp}$.

MIREAULT, Réal, L'ancienneté dans les promotions en régime de négociation collective, 1958, $112 \mathrm{pp}$.

NADEAU, Jocelyn, Les cadres de l'entreprise industrielle nord-américaine, 1960, 93 $\mathrm{pp}$.

NADON, Jacques, Centralisation et décentralisation de la fonction "Personnel», 1962, $101 \mathrm{pp}$.

NGUYEN, Van Tung, Évolution du contenu de la convention collective aux ÉtatsUnis, 1956, $132 \mathrm{pp}$.

NORBERT, GARCEAU, Denise, Une méthode de sélection basée sur les données biographiques dans la formule de demande d'emploi, 1973, 122 pp.

NORMANDIN, Robert, L'ajustement automatique des salaires, 1961, $79 \mathrm{pp}$.

O'MEARA, Raymond, La conciliation, rôle d'une optique situationnelle accommodative et normative, 1974, $132 \mathrm{pp}$.

OUELLET, Florian, Aspects ergonomiques de la compression de la semaine de travail, 1972, $273 \mathrm{pp}$.

OUELLET, Jacques, L'unité de négociations, 1956, 79 pp.

OUELLETTE, Jean-Paul, Typologie des différents rôles que peut assumer l'arbitre des griefs, 1971, $108 \mathrm{pp}$.

PANET-RAYMOND, Francyne, Le piquetage: légalité et pratique, 1968, $135 \mathrm{pp}$.

PAQUETTE, Jean, La convention collective à la Dominion Textile Co., 1961, 102 pp.

PARADIS, Claude, Le pluralisme syndical et son application aux relations patronales ouvrières de Radio-Canada, 1958, $113 \mathrm{pp}$.

PARENT, Robert, L'administration du personnel dans les hôpitaux, 1952, 112 pp. 
PAYETTE, Jean, Étude d'un conflit de travail entre cadres, à Radio-Canada, 1966, $260 \mathrm{pp}$.

PÉPIN, Fernand, La grève de l'amiante à Asbestos,

PERRAS, Sylvio, Pluralisme syndical québécois, 1955, 110 pp.

PERREAULT, Guy, Nature et facteurs de développement des bénéfices marginaux dans l'industrie manufacturière au Canada, 1964, 139 pp.

PERREAULT, Lucien, Le syndicat dans l'industrie manufacturière du tabac, 1952, $71 \mathrm{pp}$.

PERRON, Gérard, Réclamation de salaire dans l'industrie de la chaussure 1946-1947, 1948, 75 pp.

PICOTTE, Pierrette, L'évaluation des fonctions de la direction du personnel en administration publique, 1957, 89 pp.

PINEL, Marthe, L'inflation française de 1945-1951,

PITRE, Hubert, Un service du personnel dans une entreprise à contrat, 1962, $96 \mathrm{pp}$. PLANTE, Yves, La réduction du nombre des facteurs dans un système d'évaluation des emplois par points, 1971, $217 \mathrm{pp.}$

POUPART, GENDRON, Nicole, Le chômage et les taux d'activité des jeunes de 14 à 24 ans selon le statut scolaire 1966-1972, 1973, $121 \mathrm{pp}$.

PROVENÇAL, Lucien, La nouvelle carte syndicale canadienne après la fusion, 1957, 94 pp.

QUINET, Félix, Les courants catholique et socialiste dans la réforme de l'entreprise, $1955,113 \mathrm{pp}$.

RAYMOND, Jean-Guy, Le problème des loisirs et l'industrie moderne, 1954, 216 pp.

RAYNAULD, André, La grève des instituteurs, 1951, 209 pp.

RÉGIMBAL, Roger, Labour Conditions at the International Nickel Co. of Canada, Ltd., Sudbury, Ontario, 1944-1945, 1946, 43 pp.

RIVARD, J.-Claude, Étude des facteurs influant sur l'absentéisme des enseignantes de la CECM, 1969, $161 \mathrm{pp}$.

RIVEST, Gérald, La mise en marche réglementée des produits agricoles, 1960, 127 pp.

RIVEST, Jean-Louis, Étude du roulement de la main-d'oeuvre industrielle, 1946, 96 pp.

ROBERT, Marc, Mouvement général des salaires 1938-1946, 1948, 87 pp.

ROBIN, Jacquelin, Étude comparative des caractéristiques d'employés d'une fabrique d'aluminium qui ont fait des griefs, 1971, $114 \mathrm{pp}$.

RONDEAU, Claude, La politique des revenus, 1966, $237 \mathrm{pp}$.

ROUSSEAU, André, Le tribunal du travail, 1969, $169 \mathrm{pp}$.

ROUSSEAU, Clément, Le droit au travail,

ROY, Jules, Les causes du conflit de 1967 dans le monde scolaire, 1971, $160 \mathrm{pp}$.

ROY, Thérèse, Influence économique et sociale des allocations familiales, 1948, 71 pp.

SAINT-GERMAIN, Jacques, L'influence du salaire sur la satisfaction générale au travail des infirmières (milieu hospitalier québécois), 1974, 238 pp.

STE-MARIE, Michel, Analyse des heures de travail dans l'industrie manufacturière québécoise, 1974, $256 \mathrm{pp}$. 
SANSOUCY, Bernard, Le conflit industriel dans la législation du travail nordaméricaine, 1961, $118 \mathrm{pp}$.

SAVARY, Alain, La planification du personnel enseignant; application au cas de la CECM, 1970, 178 pp.

SÉNÉCAL, Yvan, L'association professionnelle des industriels, 1954, 222 pp.

SMET, Jenny, La mobilité industrielle des diplômés en relations industrielles de l'Université de Montréal, 1980, 218 pp.

SUYS, Jean-Pierre, Conversion industrielle/Droit et responsabilité de la direction, 1970, $135 \mathrm{pp}$.

TAPIN, Jean-Robert, Absentéisme du professionnel dans la fonction publique, 1973, 99 pp.

THIBAUDEAU, André, La procédure en matière de griefs, 1960, $129 \mathrm{pp}$.

THIBODEAU, Martin, Effet de restriction des régimes de retraite sur la mobilité des travailleurs, $1973,98 \mathrm{pp}$.

TOUSSAINT, Jean, Les coopératives de pêcheurs de la Gaspésie, 1948, 105 pp.

TREMBLAY, Serge, Impact de la clause de vacances des infirmières dans les hôpitaux du Québec, 1973, 201 pp.

TREMBLAY, L.G., Habitations familiales, 1949, 64 pp.

TREMBLAY, Pierre-Michel, La sécurité d'emploi comme facteur motivationnel chez les employés de langue française et de langue anglaise dans un organisme gouvernemental, 1968, $189 \mathrm{pp}$.

VADEBONCOEUR, Francine, La profession d'infirmière historique et développement du syndicalisme à Montréal,

VAILLANCOURT, Michel, Le syndicalisme des médecins au Québec, 1966, $176 \mathrm{pp}$.

VAILLANCOURT, Noëlla, Le travail des enfants et des adolescents,

VAN HOUTTE, Christian, La sécurité au travail des travailleurs dans l'industrie de la construction, $1970,163 \mathrm{pp}$.

VERDOODT, Albert, Relations entre anglophones et francophones dans les syndicats québécois, 1971, $234 \mathrm{pp}$.

VERSCHELDEN, Louise, La responsabilité civile des syndicats en cas de grève illégale, 1979, $293 \mathrm{pp}$.

VILLENEUVE, Jacques, L'apprentissage dans l'imprimerie de Montréal, 1936-1947,

VILLENEUVE, Roland, Institut d'éducation syndicale dans la province de Québec, 1949, $87 \mathrm{pp}$.

VINCENT, Janine, L'impact de variables contextuelles sur le déroulement de la négociation collective, 1979, $111 \mathrm{pp}$.

VINCENT, Roger, Les travailleurs en forêt et la Canadian International Paper Co., $1953,150 \mathrm{pp}$.

WILS, Thierry, La détermination de l'optique relationniste dans l'explication des structures salariales, $1978,225 \mathrm{pp}$.

ZRINYI, Joseph, Les conventions collectives et la confédération des travailleurs catholiques du Canada, 1953, 91 pp. 